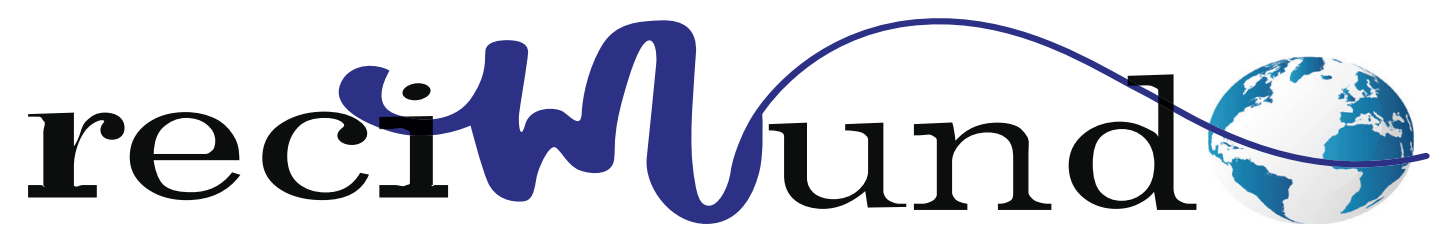

Revista Científica Mundo de la Investigación y el Conocimiento

DOI: 10.26820/recimundo/4.(4).noviembre.2020.355-363

URL: http://recimundo.com/index.php/es/article/view/959

EDITORIAL: Saberes del Conocimiento

REVISTA: RECIMUNDO

ISSN: 2588-073X

TIPO DE INVESTIGACIÓN: Artículo de revisión

CÓdIGO UNESCO: Ciencias Médicas

PAGINAS: 355-363

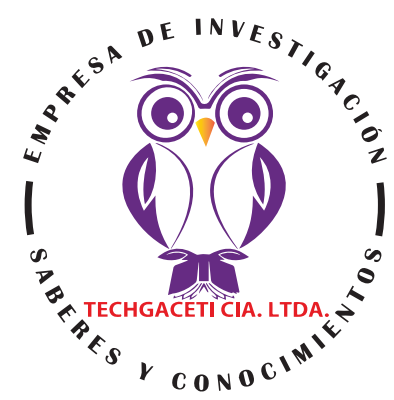

\title{
Cuidados respiratorios para el recién nacido ventilado
}

Respiratory care for the ventilated newborn

Cuidados respiratórios para o recém-nascido ventilado

Jessenia Elizabeth Carvache Zambrano ${ }^{1}$

RECIBIDO: 10/07/2020 ACEPTADO: 02/09/2020 PUBLICADO: 10/11/2020

1. Médico; Investigadora Independiente; Guayaquil, Ecuador; jessyeliza26@gmail.com; iD https://orcid.org/0000-0002-06522980

CORRESPONDENCIA

Jessenia Elizabeth Carvache Zambrano

jessyeliza26@gmail.com

Guayaquil, Ecuador

๑ ( RECIMUNDO; Editorial Saberes del Conocimiento, 2020 


\section{RESUMEN}

Todo proceso de nacimiento viene acompañado de grandes cambios fisiológicos, los cuales suelen revelar ciertas alteraciones que no representaban problema alguno a lo largo de la vida intrauterina. El Síndrome de Dificultad Respiratoria (SDR) es uno de los problemas más frecuentes que se presentan en el recién nacido. El grado de gravedad de estas enfermedades aumenta con el grado de prematurez. Las complicaciones del SDR son la hemorragia intraventricular, la lesión de la sustancia blanca periventricular, el neumotórax a tensión, la displasia broncopulmonar, la sepsis y la muerte del recién nacido. Las complicaciones intracraneales se han vinculado a hipoxemia, hipercarbia, hipotensión, fluctuaciones de la tensión arterial e hipoperfusión cerebral. Su adecuado manejo es fundamental para la supervivencia del paciente, así como evitar las complicaciones que de un mal manejo se puedan derivar. El objetivo fundamental de la presente investigación consiste en compendiar algunos aspectos importantes del cuidado básico para el recién nacido ventilado. El modelo de investigación es una revisión de tipo documental bibliográfico. Los recién nacidos con problemas respiratorios ameritan cuidados especiales, los cuales consisten básicamente en administrar surfactante intratraqueal, oxígeno suplementario y ventilación mecánica, según sea necesario. La adecuada administración de estos tratamientos en recién nacidos ventilados mejora el pronóstico de estos pacientes y evita complicaciones importantes asociadas con este manejo. Existen numerosas guías para su manejo, no obstante, el tratamiento debe ser individualizado y con prevalencia del juicio clínico.

Palabras clave: Cuidados, Respiratorios, Recién, Nacido, Ventilado.

\section{ABSTRACT}

All birth processes are accompanied by major physiological changes, which usually reveal certain alterations that do not represent a problem during intrauterine life. Respiratory Distress Syndrome (RDS) is one of the most common problems that occur in the newborn. The degree of severity of these diseases increases with the degree of prematurity. Complications of RDS include intraventricular hemorrhage, periventricular white matter injury, tension pneumothorax, bronchopulmonary dysplasia, sepsis, and death of the newborn. Intracranial complications have been linked to hypoxemia, hypercarbia, hypotension, fluctuations in blood pressure, and cerebral hypoperfusion. Its proper management is essential for the survival of the patient, as well as avoiding complications that may arise from poor management. The main objective of this research is to summarize some important aspects of basic care for the ventilated newborn. The research model is a bibliographic documentary type review. Newborns with respiratory problems require special care, which basically consists of administering intratracheal surfactant, supplemental oxygen, and mechanical ventilation, as needed. The adequate administration of these treatments in ventilated newborns improves the prognosis of these patients and avoids important complications associated with this management. There are numerous guidelines for its management, however, treatment must be individualized and with prevalence of clinical judgment.

Keywords: Care, Respiratory, Newborn, Born, Ventilated.

\section{RESUMO}

Todos os processos de nascimento são acompanhados de grandes alterações fisiológicas, que geralmente revelam certas alterações que não representam um problema durante a vida intra-uterina. A Síndrome do Desconforto Respiratório (SDR) é um dos problemas mais comuns que ocorrem no recém-nascido. O grau de gravidade destas doenças aumenta com o grau de prematuridade. As complicações da SDR incluem hemorragia intraventricular, lesão periventricular da matéria branca, pneumotórax de tensão, displasia broncopulmonar, sepsis, e morte do recém-nascido. As complicações intracranianas foram associadas à hipoxemia, hipercarbia, hipotensão, flutuações na pressão sanguínea e hipoperfusão cerebral. A sua gestão adequada é essencial para a sobrevivência do paciente, bem como para evitar complicações que possam surgir de uma má gestão. O principal objectivo desta investigação é resumir alguns aspectos importantes dos cuidados básicos para o recém-nascido ventilado. O modelo de investigação é uma revisão de tipo documental bibliográfico. Os recém-nascidos com problemas respiratórios requerem cuidados especiais, que consistem basicamente na administração de surfactante intratraqueal, oxigénio suplementar, e ventilação mecânica, conforme necessário. A administração adequada destes tratamentos em recém-nascidos ventilados melhora o prognóstico destes pacientes e evita complicações importantes associadas a esta gestão. Existem numerosas directrizes para a sua gestão, no entanto, o tratamento deve ser individualizado e com prevalência de julgamento clínico.

Palavras-chave: Cuidados, Respiratório, Recém-nascido, Nascido, Ventilado. 


\section{Introducción}

Todo proceso de nacimiento viene acompañado de grandes cambios fisiológicos, los cuales suelen revelar ciertas alteraciones que no representaban problema alguno a lo largo de la vida intrauterina. En consecuencia, todo parto requiere de personal experto en reanimación neonatal, ya que los problemas respiratorios son de los más frecuentes.

"El síndrome de dificultad respiratoria (SDR) se debe a la deficiencia de agente tensioactivo en los pulmones de los recién nacidos, más frecuentemente en los que nacen con < 37 semanas de gestación". Se estima que el riesgo aumenta con el grado de prematurez. Entre los signos y síntomas más frecuentes se tienen:

- Respiración con quejido.

- Uso de músculos accesorios.

- Aleteo nasal que aparecen poco después del nacimiento. (Lattari Balest, 2019).

Sweet et al., (2013) referido por Morales et al. (2015) refiere acerca del SDR, lo siguiente:

La incidencia de SDR se incrementa con una edad gestacional menor; en el año de 2010 el EuroNeoNet informó una incidencia del $92 \%$ en recién nacidos (RN) de 24 a 25 semanas, del $88 \%$ entre RN de 26 a 27 semanas, del $76 \%$ en prematuros de 28 a 29 semanas y del $57 \%$ en niños que nacieron entre las 30 y 31 semanas de gestación (SDG). Los estudios multicéntricos más recientes muestran que en pacientes de 26 a 29 semanas, que son manejados de forma inicial con presión positiva continua a las vías aéreas (CPAP), se reduce la necesidad de intubación o uso de surfactante hasta en un $50 \%$. (p. 169)
Entre las principales complicaciones del SDR tenemos:

- La hemorragia intraventricular

- La lesión de la sustancia blanca periventricular

- El neumotórax a tensión

- La displasia broncopulmonar

- La sepsis y la muerte del recién nacido

- Las complicaciones intracraneales vinculadas a hipoxemia, hipercarbia, hipotensión, fluctuaciones de la tensión arterial e hipoperfusión cerebral. (Lattari Balest, 2019)

Para Robaina, Riesgo, \& López (2017) la ventilación mecánica convencional (VMC) hasta los momentos sigue siendo el tratamiento primario de la insuficiencia respiratoria en el recién nacido, asimismo, la ventilación mecánica con presión positiva continúa "siendo el enfoque más común, a pesar de la introducción de nuevas estrategias como la oxigenación de membrana extracorpórea, el óxido nítrico inhalado, la ventilación de alta frecuencia, la ventilación líquida parcial y la ventilación dirigida por volumen". (p. 341)

Asimismo, el uso de tratamientos como el surfactante y el oxígeno son de vital importancia en los cuidados respiratorios para el recién nacido ventilado, ya que permiten estabilizar su condición respiratoria. No obstante, requiere un adecuado manejo que evite las complicaciones a que pueda conllevar su uso.

El objetivo de la presente investigación consiste en plasmar el uso de ventiladores, oxígeno y surfactante como parte de los cuidados respiratorios básicos para el recién nacido ventilado.

\section{Materiales y Métodos}

La presente investigación se realizó mediante una búsqueda de material bibliográfico digitalizado. Este material fue la base

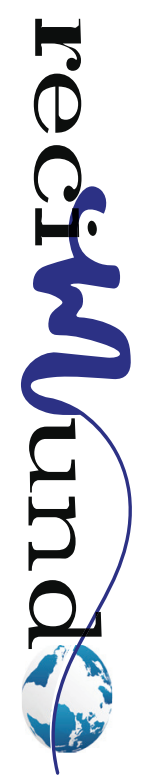


fundamental para el análisis y compendio del tema: el uso de ventiladores, oxígeno y surfactante como parte de los cuidados respiratorios básicos para el recién nacido ventilado. En razón de lo cual, la presente investigación se clasifica como de tipo documental o bibliográfica.

Se utilizaron varias bases de datos para la búsqueda entre las que destacan: SciELO, Redalyc, Medigraphic, ELSEIVER, entre otros. Asimismo, se empleó el uso de páginas web del área de la salud con reconocimiento científico mundial tales como: Organización Mundial de la Salud (OMS), Organización panamericana de la Salud (OPS) y Manuales MSD.

Para el tipo de material fueron seleccionados informes, protocolos, tesis de grado y otras clases de contenidos. Se excluyeron los contenidos repetidos, las editoriales 0 cartas editoriales, anotaciones académicas y todo documento carente de sustento científico o bajo nivel de evidencia.

En cuanto a los descriptores, fueron usaron los siguientes: "cuidados respiratorios para el recién nacido", "manejo del recién nacido ventilado"; "ventilación en recién nacidos", "oxigeno de suplantación en recién nacidos", "surfactante en el tratamiento de enfermedades respiratorias en recién nacidos" y "síndrome de dificultad respiratoria". La información encontrada fue filtrada bajo los criterios de idioma español, mayor relevancia y correlación temática. Igualmente, se seleccionaron aquellos trabajos de mayor actualidad (fecha de publicación de 09 años). La investigación se llevó a cabo durante el mes de octubre del presente año.

\section{Resultados}

\section{Generalidades}

Para Villegas Silva, (2016) los avances tecnológicos han permito mejorar paulatinamente la ventilación mecánica, llevándola no solo a suplir los esfuerzos de los recién nacidos y niños para su ventilación, sino colaborando en la disminución del trabajo respiratorio, además de permitir una mayor adaptación. La presión positiva continua de vías aéreas (CPAP; por sus siglas en inglés), se usa más tempranamente, ya que permite una mejor salida del líquido pulmonar y más rápida formación de la capacidad funcional residual (CFR). En consecuencia, siempre que el niño tenga una respiración espontánea, puede sostenerse con este método no invasivo de apoyo a la ventilación. (p. 11)

Asimismo, estos cuidados respiratorios habituales para el recién nacido ventilado incluyen suplementos de oxígeno y surfactantes.

\section{Surfactante}

"El tratamiento específico del SDR se realiza con agente tensioactivo intratraqueal. Este tratamiento exige intubación endotraqueal, que también puede ser necesaria para lograr ventilación y oxigenación adecuadas". (Lattari Balest, 2019)

La distensibilidad se basa en la recuperación elástica de los tejidos y del surfactante exógeno. Por eso al hablar de mecánica de la ventilación en recién nacidos es indispensable tomar en cuenta al surfactante. Este es un complejo molecular formado en los neumocitos tipo II alveolares y que puede estar presente desde las semanas 24 a 25 de la gestación, está formado principalmente por fosfolípidos y de estos la fosfatidilcolina es el principal. Tiene además proteínas que dan una estructura química específica, permite su estabilización en la luz del alveolo y formación de una capa adecuada en este sitio, además estas proteínas tienen una función inmunológica, tiene además grasas neutras. (Villegas Silva, 2016, p. 13)

Para (Organización Panamericana de la Salud/Organización Mundial de la Salud (OPS/ OMS) - Representación Guatemala, 2015) 
En el Síndrome de Dificultad Respiratoria (SDR) (Enfermedad de Membrana Hialina) el tratamiento con surfactante se emplea en los siguientes casos:

1. Necesidad de $\mathrm{FiO} 2>30$ para mantener una $\mathrm{PaO} 2$ arterial $>80$ torr $\mathrm{y} / \mathrm{o} \mathrm{SaO} 2>90 \%$ y/o FiO2 $>30$ asociado con FR >60 rpm. a pesar de intubación, CPAP ó VMI. 2. Survanta (natural): $4 \mathrm{ml} / \mathrm{kg}$ por dosis, ET (viales de $8 \mathrm{ml}$, usar una sola vez; mantener en refrigeración de $2^{\circ} \mathrm{C}$ a $8^{\circ} \mathrm{C}$ ). 3. Exosurf (sintéti$\mathrm{co}$ ): $5 \mathrm{ml} / \mathrm{kg}$ por dosis, ET (viales de 7.5 a 8 $\mathrm{ml}$. La suspensión reconstituida es estable por 12 horas almacenada de $2^{\circ} \mathrm{C}$ a $30^{\circ} \mathrm{C}$ ).

\section{En cuanto a su forma de aplicación, los mismos autores refieren:}

No se requiere mover al neonato para asegurar una buena distribución ya que puede causar más deterioro. En general la administración rápida es mejor. Sin embargo, en neonatos muy inestables es mejor administrarlo en un período de 15 a 30 minutos. No succionar durante dos horas después de la administración. La sedación rutinaria no es recomendada. En casos en que no se logre una buena sincronización con la VMI y la respiᄀración, puede utilizarse la parálisis con: Pancuronio: $0.1 \mathrm{mg} / \mathrm{kg}$, IV en infusión directa, o Vecuronio: $0.1 \mathrm{mg} / \mathrm{kg}$, IV en infusión directa. Para sedación y analgesia puede utilizarse: Fentanil: 0.5 a $4 \mu \mathrm{g} / \mathrm{kg} / \mathrm{do}-$ sis, IV en infusión lenta, o Midazolam: 0.05 a $0.15 \mathrm{mg} / \mathrm{kg}$ en infusión lenta por 5 minutos, o Morfina: 0.05 a $0.2 \mathrm{mg} / \mathrm{kg}$ por dosis, IV infusión lenta en 5 minutos. Se puede repetir la dosis cada 4 horas. En cuanto al monitoreo se debe mantener gases arteriales en valores relativamente normales: $\mathrm{PaO} 2: 60-90$ mmHg, PaCO2: 35 a 50 mm Hg y pH: 7.30 a 7.45. Monitoreo continuo de la $\mathrm{SaO} 2$ a través de un oxímetro de pulso. Respuesta a la ventilación de acuerdo a: Movimientos torácicos, entrada de aire, color, $\mathrm{PaO} 2, \mathrm{SaO} 2$, $\mathrm{PaCO} 2, \mathrm{FiO} 2$ y presiones de ventilación $\mathrm{FC}$ y PA. Vigilar por barotrauma: Neumotórax, neumomediastino, neumopericardio o neu- moperitoneo y enfisema intersticial. Es importante realizar un seguimiento debido a que la toxicidad del oxígeno puede resultar en enfermedad pulmonar crónica, displasia broncopulmonar o retinopatía. Asimismo, la hipoxemia y la acidosis pueden resultar en secuelas neurológicas severas. (p. 269, 270)

\section{Oxígeno}

En cuanto al oxígeno suplementario, es importante considerar que los prematuros con SDR una presión suficiente para la apertura de su vía respiratoria, así como mejorar la capacidad funcional residual (CFR). Con esto se quiere disminuir el esfuerzo respiratorio necesario y mejorar la oxigenación. El oxígeno solo se aporta según la necesidad de cada paciente, por cuanto se cubrió la presión necesaria. (Villegas Silva, 2016, p. 13)

Para Castro et al. (2011), en recién nacidos de término que requieren reanimación con ventilación a presión positiva (VPP), el uso al inicio de $\mathrm{O} 2$ al 1,0 no ofrece ventajas sobre el uso de aire ambiente y requiere mayor tiempo hasta lograr la primera respiración espontánea o llanto. Varios estudios en recién nacidos han demostrado que la presencia de estrés oxidativo con la re-oxigenación causa daño al corazón, riñón y un estado metabólico pro-oxidante que persiste más allá del mes de nacimiento. Recientes estudios apoyan las siguientes recomendaciones:

- El uso de aire como gas inicial en la reanimación del recién nacido de término

- El uso de la oximetría de pulso como un método factible y fiable para el control de suplemento de oxígeno en la sala de partos

- El inicio de la reanimación con bajas concentraciones de oxígeno ( $\mathrm{FiO} 2$ 0,30$0,50)$ en recién nacidos prematuros

- Si a pesar de una ventilación eficaz no hay un aumento en la frecuencia cardía- 
ca o si la oxigenación (guiados por oximetría) sigue siendo inaceptable, debe considerarse el uso de una mayor concentración de oxígeno. (p. 537, 538)

El Ministerio de Salud de Uruguay (2019), respecto a los cuidados respiratorios para el recién nacido prematuro destacan la importancia de evitar la hipoxia e hiperoxia durante la asistencia en sala de partos. Asimismo, refieren las siguientes generalidades en cuanto a la oxigenación del recién nacido ventilado:

Los sistemas antioxidantes en recién nacidos prematuros son deficientes y la toxicidad del oxígeno puede contribuir a aumentar el riesgo de enfermedad respiratoria crónica, retinopatía del prematuro, hemorragia intraventricular y enterocolitis necrotizante. El aporte de oxígeno debe ser controlado desde los primeros minutos de vida y la dosis debe ser adecuada a las necesidades del paciente. En 2015 las guías de resucitación internacionales recomendaron fuertemente, en recién nacidos con edad gestacional $\leq 35$ semanas, iniciar la estabilización con una FiO2 entre $0.21-0.30$ y no mayor de 0.65. Sin embargo, estudios recientes han puesto en duda estas recomendaciones, al informar mayor mortalidad o mayor mortalidad e injuria cerebral en los grupos de pacientes que recibieron menor concentración de $\mathrm{O} 2$ en la asistencia inicial (FiO2 0.21 o titulación de la FiO2 desde 0.21). Para administrar la concentración de $\mathrm{O} 2$ que el paciente requiere es necesario contar en la sala de nacimientos con un blender o mezclador aire/oxigeno, esta es una recomendación de las guías internacionales desde el 2010. Asimismo, los autores recomiendan para la administración de O2:

- Aplicar FiO2 0.30 al inicio de la estabilización y titularla de acuerdo a la saturometría de pulso parece ser una conducta adecuada de acuerdo a la información disponible.

- No se recomienda administrar $\mathrm{FiO} 2$ >
0.65 en el inicio de la asistencia en recién nacidos prematuros. (p.60)

\section{Ventilación}

Según la Guía para el manejo integral del recién nacido grave de la Organización Panamericana de la Salud y la Organización Mundial de la Salud (OPS/OMS) - Representación Guatemala (2015), existen los siguientes tipos de ventiladores:

- Ventilador de presión: entregan un flujo de gas continuo, precalentado y humidificado a una presión inspiratoria y final espiratoria predeterminada (por ejemplo: Baby Bird, Bear Cub, Infant Star, Sechrist, Dragger, Healthdyne)

- Ventilador de volumen: se preselecciona el volumen de gas a entregar al sistema (por ejemplo: Benett MA1 y 2, Bour $\neg$ nes 104-50, Bird VIP, Newport Breeze, Siemens Servo 300)

- Ventilador de alta frecuencia: entregan volúmenes tidales (corrientes) menores que el espacio muerto anatómico, con frecuencias elevadas. Existen dos tipos: Ventilador jet de alta frecuencia (HFJV), entrega pulsos cortos de gas a las vías aéreas superiores, con frecuencias desde 140 hasta 600/min. La exhalación es pasiva y depende de la reacción pulmonar.

- Ventilación oscilatoria de alta frecuencia (HFOV), entrega un flujo de gas continuo a una presión media constante. La presión oscilatoria produce alrededor de la vía aérea pequeños volúmenes tidales (corrientes) a un volumen pulmonar medio constante. La exhalación es activa lo cual facilita la salida de CO2. (p. 304)

Por otra parte, Ferrer, Cuesta, Rodríguez, \& Estévez (2012) señalan como principales causas de asistencia ventilatoria las afecciones respiratorias (enfermedad de la membrana hialina y la neumonía de debut 
temprano). Con respecto a la edad para iniciar la ventilación mecánica, algunos autores coinciden en que mientras más precoz se realice, se obtendrán mejores beneficios y menores complicaciones. Asimismo, esta ventilación en edad temprana produce una menor estadía en la ventilación y una mayor supervivencia. (p. 195)

Castillo et al., (20517) con relación a la Ventilación de Alta Frecuencia (VAF), refiere que la principal indicación es la necesidad de reclutamiento pulmonar. En ella se propone el open lung, que consiste en el uso de una presión media en la vía aérea (MAP) que sea suficiente para permitir el máximo reclutamiento alveolar impidiendo la atelectasia. El consenso clínico consideraría el uso de esta modalidad en las siguientes situaciones:

1. Durante la ventilación mecánica convencional cuando se necesite presiones inspiratorias pico mayores de $25 \mathrm{~cm} \mathrm{H} 2 \mathrm{O}$ para conseguir una adecuada ventilación.

2. Cuando es necesario suplementar oxígeno por encima de 0,6 tras optimizar la ventilación mecánica convencional con signos de sobre distensión (curvas presión-volumen, C20/C <0,8).

3. Fuga aérea.

Asimismo, estos autores refieren en cuanto al inicio y mantenimiento de la VAF:

- Oxigenación. En el caso del uso de VAF debido a fuga aérea se iniciará con la misma Presión de distensión continua de la vía aérea (PDC) que en ventilación mecánica convencional y se mantendrá una actitud más conservadora tolerando FiO2y pCO2 más altas. No todos los ventiladores de alta frecuencia producen la misma hiperinsuflación con la misma presión media en la vía aérea (MAP). Además, se ha de tener en cuenta otros signos de hiperinsuflación, como el aplanamiento diafragmático o la compresión de la silueta cardiaca.

- Ventilación. La amplitud inicial se situará en entre un 40-50\% (dependerá del tipo de respirador que se use) y se irá ajustando en intervalos del 10\% para mantener un volumen corriente entre 1,5-2 cc/ $\mathrm{kg}$ y/o una pCO2 adecuada para cada paciente. La frecuencia respiratoria (FR) se pautará en relación con el peso del paciente: en menores de $1.500 \mathrm{~g}$ pueden usarse FR de 12-15 Hz, mientras que en prematuros tardíos o recién nacidos a término pueden manejarse alrededor de $10 \mathrm{~Hz}$. Pueden ser necesarias FR más bajas en caso de enfermedad pulmonar grave. (p. 295.e4)

El destete o desconexión del ventilador es sumamente importante. Se puede desconectar del ventilador al recién nacido cuando el estado respiratorio de este mejore. Este puede desconectarse del ventilador (destete) por medio del descenso de FIO2, presión inspiratoria y frecuencia. "A medida que se reduce la tasa, el niño adquiere mayor capacidad de trabajo respiratorio. Los lactantes que pueden mantener la oxigenación y la ventilación adecuada en los ajustes más bajos suelen tolerar la extubación". Los últimos pasos para la retirada gradual del ventilador son:

- Extubación

- Posible soporte con CPAP nasal (o nasofaríngeo) o ventilación con presión positiva no invasiva

- Uso de una capucha o una cánula nasal para suministrar oxígeno o aire humidificado. (Lattari Balest, 2019)

\section{Complicaciones}

Las complicaciones más frecuentes de la ventilación mecánica entre los recién nacidos son:

- Neumotórax

- Asfixia por obstrucción del tubo endotraqueal 
- Ulceración, erosión o estenosis de las estructuras de las vías aéreas por la presión generada por el equipamiento adyacente

- Displasia broncopulmonar. (Lattari Balest, 2019)

Más ampliamente, Villegas Silva, (2016) en cuanto a las complicaciones del paciente ventilado, explica:

En cualquiera de las modalidades de ventilación se debe hacer cuidado especial de las complicaciones inmediatas, mediatas y tardías. Tratar de prevenir estas complicaciones debe ser uno de los objetivos iniciales. Por lo que desde que se inicia el apoyo en cualquiera de las modalidades debe considerarse un plan de retiro, incluyendo el suplemento de oxígeno. Las complicaciones más frecuentes en la asistencia mecánica a la ventilación están: barotrauma, volutrauma, neumonía, disminución del gasto cardiaco, lesión de la vía aérea, hemorragia pulmonar, obstrucción de cánula. Estas complicaciones han demostrado que el principal factor de riesgo es la edad gestacional y peso, entre menor sean estos los riesgos aumentan. Las diferentes técnicas de apoyo a la ventilación tratan de disminuir estas complicaciones, haciendo más amigables las curvas de presión volumen que se desprenden al paciente, equipos más sensibles y con más alarmas. Pero hasta ahora seguimos dependiendo en la mayoría de los caos de una vigilancia cuidadosa de nuestros equipos de enfermería y médicos encargados de las $\mathrm{UCl}$, quienes deben tener un conocimiento amplio de las variables fisiológicas del paciente y los cambios que se tienen en relación con las complicaciones, que se pueden identificar tempranamente y tratarse con oportunidad. (Villegas Silva, 2016, p. 14)

\section{Conclusión}

Uno de los principales problemas del recién nacido, es el síndrome de dificultad respi- ratoria (SDR). Estas enfermedades suelen presentarse en mayor grado cuando menor sea el grado de prematurez. Estos pacientes ameritan cuidados respiratorios especiales, los cuales consisten básicamente en administrar surfactante intratraqueal, oxígeno suplementario y ventilación mecánica, según sea necesario.

La adecuada administración de estos tratamientos en recién nacidos ventilados mejora el pronóstico de estos pacientes y evita complicaciones importantes asociadas con este manejo. Existen numerosas guías para el manejo de estos pacientes, no obstante, el tratamiento debe ser individualizado y con prevalencia del juicio clínico.

\section{Bibliografía}

Castillo, F., Fernández, D., Gutierrez, A., Moreno, J., Bustos, G., Gresa, M., \& Miracle, X. (2017). Recomendaciones para la asistencia respiratoria en el recién nacido (IV). Ventilación de alta frecuencia, ex-utero intrapartum treatment (EXIT), oxigenador de membrana extracorpórea (ECMO). (S. E. Neonatología, Ed.) Anales de Pediatría, 87(5), 295. e1-295.e7. doi:http://dx.doi.org/10.1016/j.anpedi.2017.04.003

Castillo, F., Fernández, D., Gutierrez, A., Moreno, J., Bustos, G., Gresa, M., \& Miracle, X. (20517). Recomendaciones para la asistencia respiratoria en el recién nacido (IV). Ventilación de alta frecuencia, ex-utero intrapartum treatment (EXIT), oxigenador de membrana extracorpórea (ECMO). (S. E. Neonatología, Ed.) Anales de Pediatría, 87(5), 295. e1-295.e7. doi:http://dx.doi.org/10.1016/j.anpedi.2017.04.003

Castro, A., Rabasa, C., Capellia, C., Cores, F., Enríqueza, D., Gutiérrez, S., . . . Szylda, E. (2011). Recomendaciones en Reanimación Neonatal 2011. $2^{a}$ parte: Administración de oxígeno. Estrategias ventilatorias. Masaje cardíaco. (S. A. Pediatría, Ed.) Archivos Argentinos de Pediatría, 109(6), 536-544. Recuperado el 30 de Octubre de 2020, de https://www.sap.org.ar/docs/publicaciones/archivosarg/2011/v109n6a19.pdf

Ferrer, R., Cuesta, Y. L., Rodríguez, F., \& Estévez, M. C. (2012). Superviven cia del recién nacido ventilado. Archivo Médico de Camaguey, 8(2), 190-198. Recuperado el 22 de Octubre de 2020, de http:// scielo.sld.cu/pdf/amc/v16n2/amc080212.pdf

Lattari Balest, A. (Octubre de 2019). Manuales MSD. 
Recuperado el 10 de Octubre de 2020, de https:// www.msdmanuals.com/es/professional/pediatr\%C3\%ADa/problemas-respiratorios-en-reci\%C3\%A9n-nacidos/s\%C3\%ADndrome-de-dificultad-respiratoria-en-reci\%C3\%A9n-nacidos

Lattari Balest, A. (Octubre de 2019). Manuales MSD. Recuperado el 29 de Octubre de 2020, de https:// www.msdmanuals.com/es/professional/pediatr\%C3\%ADa/problemas-respiratorios-en-reci\%C3\%A9n-nacidos/apoyo-respiratorio-en-reci\%C3\%A9n-nacidos-y-lactantes

Ministerio de Salud de Uruguay. (2019). Recomendaciones para la asistencia del recién nacido prematuro. Ministerio de la Salud de Uruguay. Organización Panamericana de la Salus - OPS. Recuperado el 29 de Octubre de 2020, de https:// www. paho.org/uru/index.php?option=com_docman\&view=download\&slug=recomendaciones-para-la-asistencia-del-recien-nacido-prematuro-web\&ltemid=307

Morales, D., Reina, E., Cordero, G., Arreola, G., Flores, J., Valencia, C., . . Villegas, R. (2015). Protocolo clínico de atención en el recién nacido con síndrome de dificultad respiratoria. Perinatología y Reproducción Humana, 29(4), 168-179. doi:http:// dx.doi.org/10.1016/j.rprh.2016.02.005
Organización Panamericana de la Salud/Organización Mundial de la Salud (OPS/OMS) - Representación Guatemala. (2015). Organización Panamericana de la Salud - OPS. Recuperado el 18 de Octubre de 2020, de https://www.paho. org/gut/index.php?option=com_docman\&view=download\&alias=773-guia-para-el-manejo-integral-del-recien-nacido-grave\&category_slug=boletines-en-web\& Itemid $=518$

Robaina, G. R., Riesgo, S. d., \& López, M. A. (2017). Ventilación mecánica en recién nacidos menores de 1500 gramos, resultados según modos de ventilación. Revista Cubana de Pediatría, 89(3), 340354. Recuperado el 28 de Octubre de 2020, de https://relaped.com/wp-content/uploads/2019/09/ GERARDO.pdf

Villegas Silva, R. (2016). Principios de fisiología y mecánica. En F. N. C., PAC Neonatología 4. Programa de actualización continua en neonatología (Vol. Libro 2, pág. 142). México D.C.: Intersistemas S.A. de C.V. Recuperado el 05 de Octubre de 2020, de https://www.anmm.org.mx/publicaciones/PAC/ PAC_Neonato_4_L2_edited.pdf

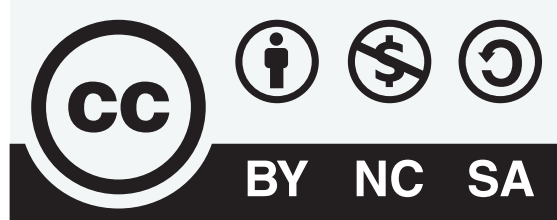 \\ CREATIVE COMMONS RECONOCIMIENTO-NOCO- MERCIAL-COMPARTIRIGUAL 4.0 .}

\section{CITAR ESTE ARTICULO:}

Carvache Zambrano, J. E. (2020). Cuidados respiratorios para el recién nacido ventilado. RECIMUNDO, 4(4), 355-363. https://doi.org/10.26820/recimundo/4. (4).noviembre.2020.355-363 